\title{
Association Analysis of Supermarket Products Based on RapidMiner
}

Heyuan Polytechnic, China

Dongxiu Ye

Yedongxiu1988@163.com

\begin{abstract}
:
With the development of information technology and database technology, information data has shown explosive growth. However, in the face of huge amounts of data, people are often confused and unable to discover the hidden knowledge and rules of the data, resulting in a waste of resources. This article uses the RapidMiner tool to analyze the data of the supermarket, analyze the products, and give examples of modeling and marketing suggestions, which has strong practical value.
\end{abstract}

Keywords: association rules, bundle sale, supermarket, marketing

\section{基于 RapidMiner 的超市商品的关联分析}

\author{
叶冬秀
}

河源职业技术学院, 河源, 广东, 中国

Yedongxiu1988@163.com

中文摘要:

随着信息技术和数据库技术的发展, 信息数据呈现爆炸式增长。然而面对海量的数据, 人们往往无所 适从，无法发现数据隐藏的知识与规则，从而导致资源的浪费。本文运用 RapidMiner 工具，分析了超 市的数据, 对产品进行关联分析, 并给出建模和营销建议的实例, 具有很强的实用价值。

关键词：关联规则 捆绑销售 超市 市场营销

\section{1. 引言}

数据挖掘 (Data Mining), 又称数据库中的知识发 现 (Knowledge Discovery in Database) [1], 顾名思义就 是从大量的数据中挖掘出有用的信息。在最近几年里已 被数据库界所广泛研究, 其中关联规则 (Association Rules) 的挖掘是一个重要的问题。数据挖掘随着计算机 应用的越来越广泛, 每年都要积累大量的数据, 运用数据 挖掘技术, 在这些数据当中我们可以找出金子来 [2]。本 文基于商家收集到顾客购买情况的数据, 使用 RapidMiner 工具, 来分析客户的购买行为及购买模式, 挖掘客户购买产品的关联数据。本文的研究不仅验证了 相关模型的可行性和准确性, 也是对关联分析理论在超 市数据中应用的一种重要探索, 具有一定的理论意义和 现实意义[3]。

\section{RapidMiner 简介及为什么选择 RapidMiner?}

\subsection{RapidMiner 简介}

Rapidminer 是世界领先的数据挖掘解决方案和完 善的商业分析平台, 是进行数据挖掘、文本挖掘和预测 分析的强大工具。它提供大量各种各样的描述性技术和 预测性技术, 能简化数据挖掘过程的设计和评价, 能从 数据中能洞察商机, 帮助做出盈利的决策。

\section{2 为什么选择 RapidMiner}

RapidMiner 不需要软件许可证费用, 是中小型企业 买得起的灵活的服务支持选项。另外, RapidMiner 还拥 有最快的开发过程 (即使针对非常复杂的数据挖掘流程) 和操作可靠性的保证, 被广泛认为是市场上最容易理解 
扩展包。它们都在 Rapid-I 市集公开发布。该市集是开 源扩展和第三方私有扩展的发布平台。

\section{3. 基于 RapidMiner 实现超市商品购买关联 分析}

个分析部件、每个预测部件，甚至报表中的每个显示部 件都可以集成到一个进程中。这意味着所有任务都可以 在一个工具中实现。更大的好处是部件之间可以互相交 互, 能够通过几次鼠标的点击集成到一起。即使对于复 杂的元素间很多交互的商业流程, 我们都可以借助 RapidMiner 简单地创建它而不需要编写任何代码; 优 势二: 前所未有的分析方便性和用户支持。依赖于元数 据的管理和进程设计的智能分析, RapidMiner 紧密关注 数据分析员的工作, 并提供实时的帮助; 优势三: 无与 伦比的分析技术的集成套装。RapidMiner 提供超过 1500 种关于数据集成、数据转换、分析、建模和可视化的方 法。市场上没有其他产品能够提供如此多的方法来定义 最优的分析进程。尤其是在属性选择和异常检测方面, 市场上其他产品都不能提供 RapidMiner 提供的大部分 方法; 优势四: 兼容主流标准。RapidMiner 支持多个标 准, 包括 PMML (预测模型标记语言), 其允许 RapidMiner 与其他系统交换预测模型。优势五: 丰富的 扩展支持。Rapid-I 和第三方提供了大量的 RapidMiner

\section{1 商业理解}

本文通过某超市在营业中收银机保存的顾客购买 记录, 来分析顾客购买的商品之间的关联性, 从而为超 市制定有效的营销策略提供依据。本文用 RapidMiner 工具制作了一个关联规则挖掘在零售业中的具体应用 模型, 这个模型主要引入了关联规则所获利润的概念讨 论超市商品捆绑销售策略[4]。

\section{2 数据理解}

本文的数据是某超市在某段时间, 1000 位顾客的购 买清单。每位顾客都购买了 7 样产品, 共 7000 条数据。

表 1 顾客购买清单

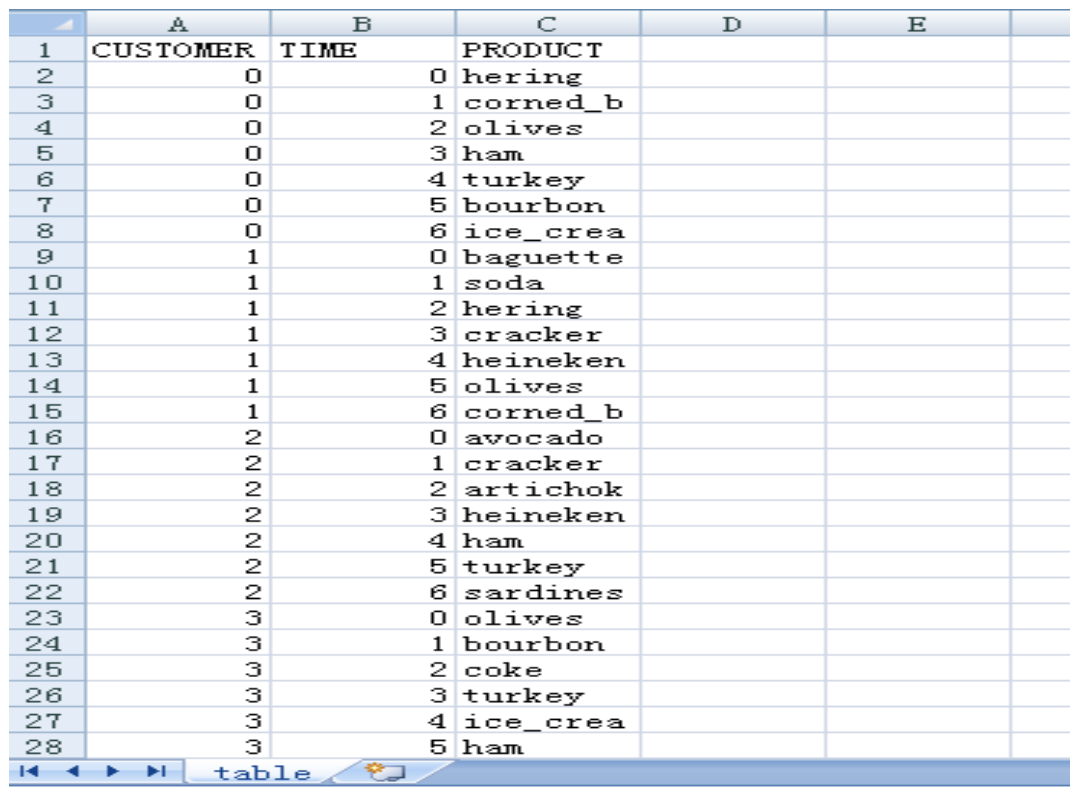

\section{3 数据准备}

每一行中: 记录该顾客购买各个商品的情况。

\section{4 建立模型与模型评估}

首先由 Read Excel 读入超市客户购买清单 Excel 表, 步骤如下: 


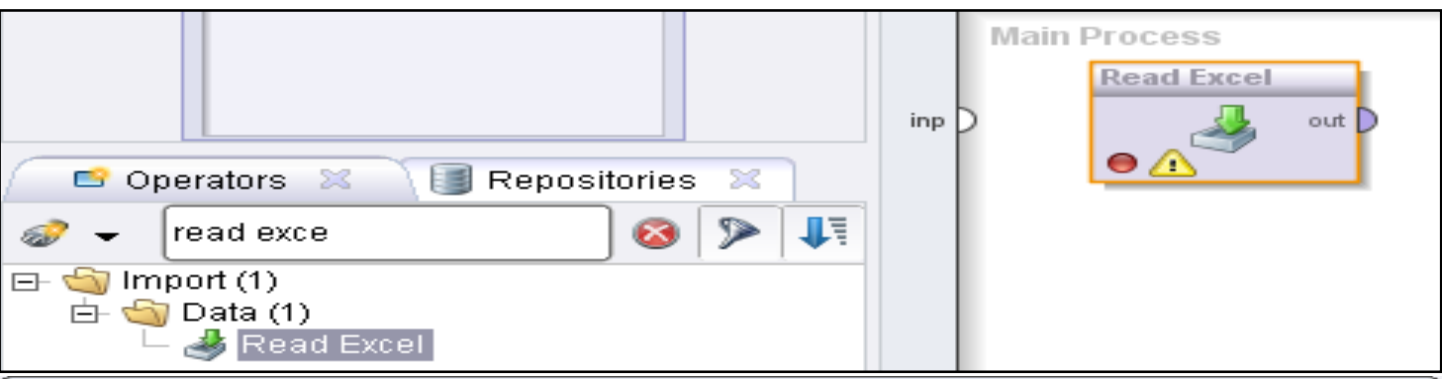

3 Data import wizard - Step 1 of 4

This wizard guides you to import your data.

Step 1: Please select the file that should be imported.

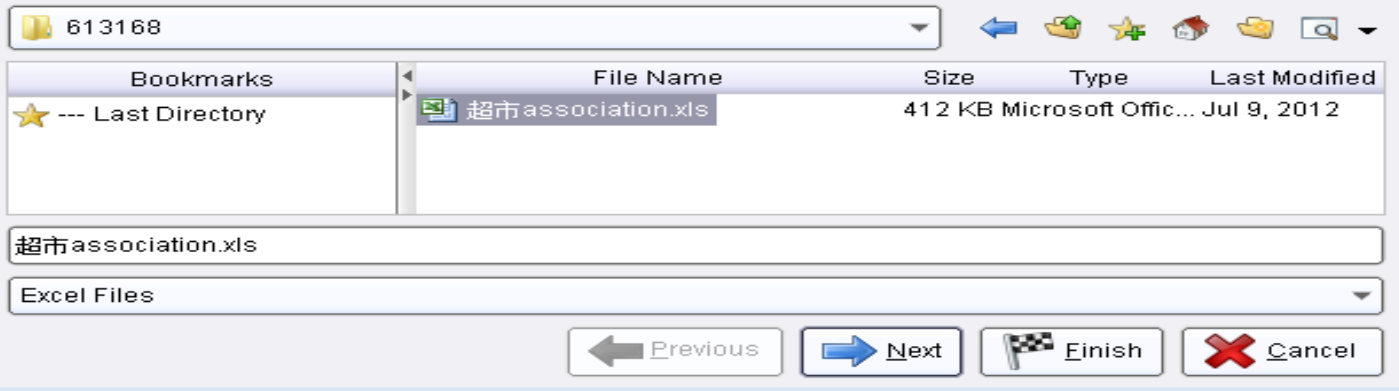

37 Data import wizard - Step 2 of 4

This wizard guides you to import your data.

Step 2: An Excel file can contain multiple sheets. Please select the one you want to import into

Rapidminer. Furthermore, you can mark a range of cells to be loaded.

\begin{tabular}{|c|c|c|}
\hline A & $\mathrm{B}$ & c \\
\hline CUSTOMER & TIME & PRODUCT \\
\hline 0 & 0 & hering \\
\hline 0 & 1 & corned_b \\
\hline 0 & 2 & olives \\
\hline 0 & 3 & ham \\
\hline 0 & 4 & turkey \\
\hline 0 & 5 & bourbon \\
\hline
\end{tabular}

Previous

\section{$\rightleftarrows$ Next}

This wizard guides you to import your data

Step 3: In RapidMiner, each attribute can be annotated. The most important annotation of an attribute is its name. If annotations are contained in the rows of your data file, you can assign them here.

\begin{tabular}{l|l|l|l|}
\hline \multicolumn{1}{|c|}{ Annotation } & \multicolumn{2}{c}{ A } & \multicolumn{1}{c|}{ B } \\
\hline Name & CUSTOMER & TIME & PRODUCT \\
\hline- & 0 & 0 & hering \\
\hline- & 0 & 1 & corned_b \\
\hline- & 0 & 2 & olives \\
\hline- & 0 & 3 & ham \\
\hline- & 0 & 4 & turkey \\
\hline- & 0 & 5 & bourbon \\
\hline- & 0 & 6 & ice crea \\
\hline
\end{tabular}




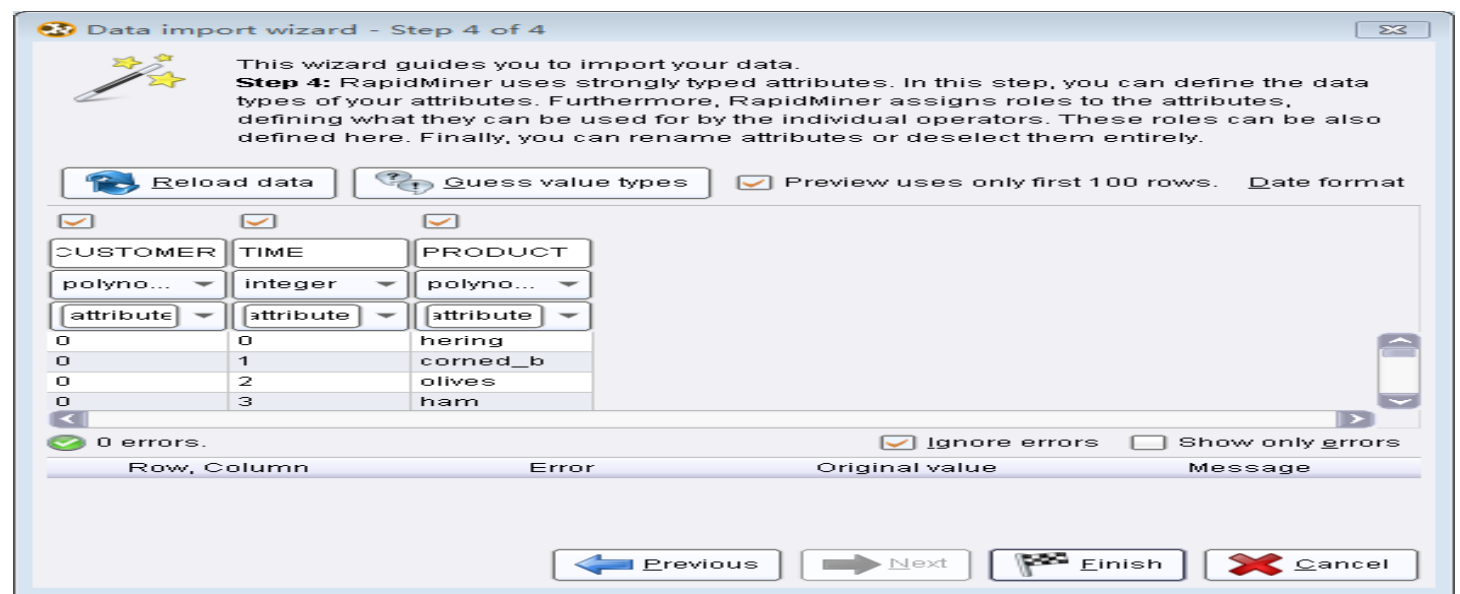

注意：第四步的 CUSTOMER 中的类型应改为 polynominal。

其次, 引入 Nominal to Binominal with Aggregation 1) 将 attribute to be converted 设成“PRODUCT”2) 勾选 "use underscore in name" 3) 点击 attributes for making groups 添加 “CUSTOMER”, 因此, 把 Excel 中的 PRODUCT 转成购买标志。操作流程如下。

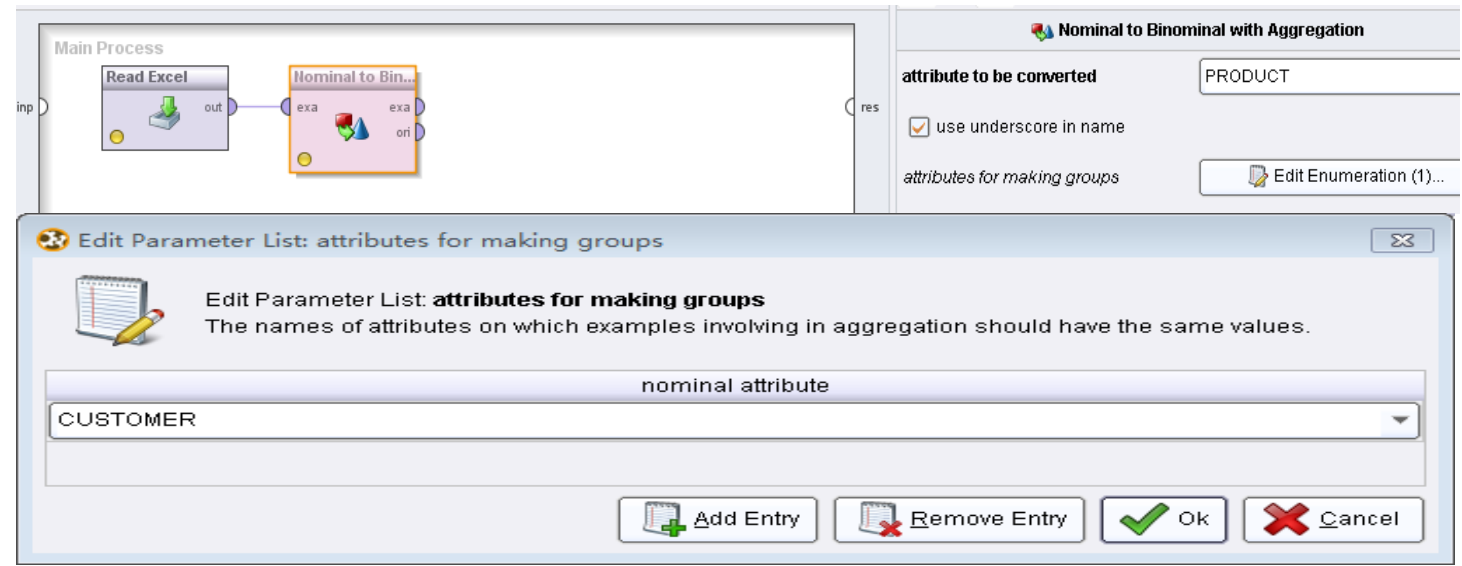

再次, 引入部件 set role, 1) 将 name 设成 “CUSTOMER"; 2) 从 target role 设成“id"; 该部件将
CUSTOMER 转变成非常规属性, 不参与后续关联分析 过程, 注意参与关联分析的属性都是常规属性。

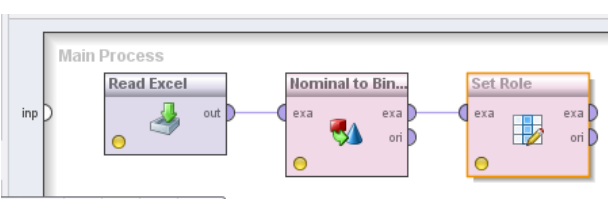

另外,引入部件 Apriori (Flat Data), 1) 勾选 find min number of itemsets; 2) 将 min support 设成 0.95 。操作

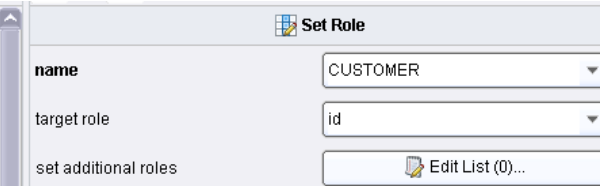

图如下。

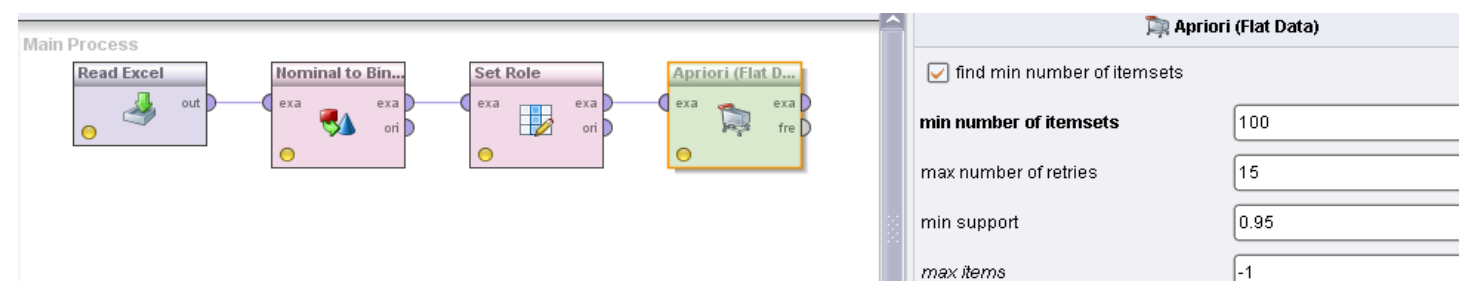


2) 将 min confidence 设成 0.8 , 该部件产生关联规则 (置

最后，为了产生关联规则，引入部件 Create Association Rules, 1) 从 criterion 中选择 confidence;
信度阈值为 $80 \%$ )。图如下。

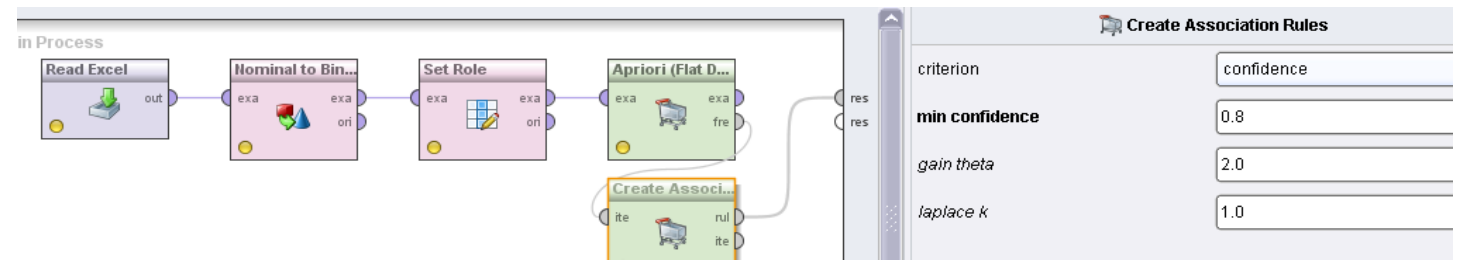

运行: 把各个部件联系起来, 并按运行键, 就出现

结果产生了四条关联规则: 了运行结果。

\begin{tabular}{|c|c|c|c|c|c|c|c|c|c|}
\hline No. & Premises & Conclusion & Suppo & Confide & LaPla.. & Gain & $p-s$ & Lift & Convi... \\
\hline 1 & PRODUCT_soda & PRODUCT_heineken & 0.257 & 0.808 & 0.954 & -0.379 & 0.066 & 1.348 & 2.088 \\
\hline 2 & PRODUCT_artichok & PRODUCT_heineken & 0.252 & 0.826 & 0.959 & -0.358 & 0.069 & 1.378 & 2.305 \\
\hline 3 & PRODUCT_heineken,PRODUCT_soda & PRODUCT_cracker & 0.234 & 0.911 & 0.982 & -0.280 & 0.109 & 1.868 & 5.726 \\
\hline 4 & PRODUCT_cracker, PRODUCT_soda & PRODUCT_heineken & 0.234 & 0.932 & 0.986 & -0.268 & 0.083 & 1.555 & 5.915 \\
\hline
\end{tabular}

结果说明:

以 NO4 为例, 对于已经买了饼干和汽水的顾客来 说, 他们就很有可能购买喜力啤酒。因为从分析结果来 看, 购买了饼干和汽水的顾客当中, 有 $93.2 \%$ (Confidence) 的顾客也同时购买了喜力啤酒; 同时购买了饼干、汽水 和喜力啤酒这三种商品的顾客占比为 $23.4 \%$ (Support)。 那么, 采用该规则向客户推荐喜力啤酒比随机推荐喜力 啤酒会提升效果 1.555 倍 (Lift)。

\section{5 模型应用}

（1）产生推荐规则并保存

由上, 已经得出了推荐规则 (即关联规则), 下面 只要引进一个Write 部件, 就可以把推荐规则保存下来。 Write 将 object file 设成“超市关联规则”文件名, 并保存 关联规则集合到文件中。图如下。

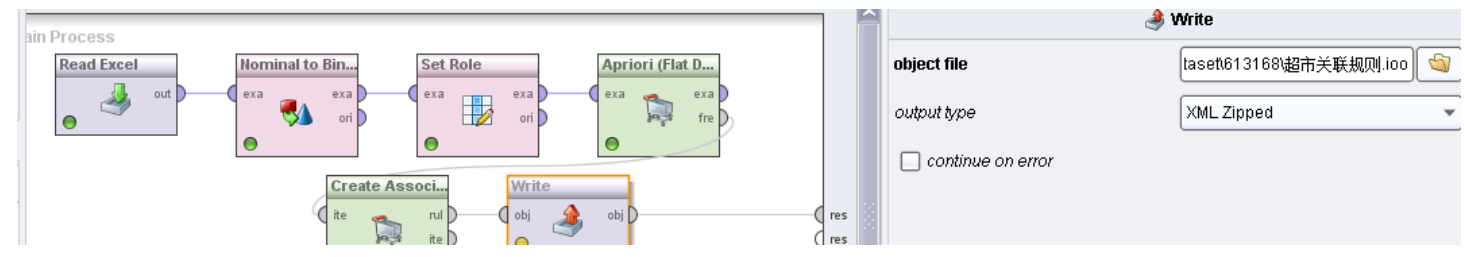

(2) 对关联性强的商品做商品推荐

首先，引入 Read Excel、Nominal to Binominal with Aggregation 和 Set role 部件, 步骤同上。
其次, 引入 Read, 1) 将 object file 设成“超市关联 规则”文件名;2）从 io object 中选择 Association Rules; 读取已经保存的关联规则集合。

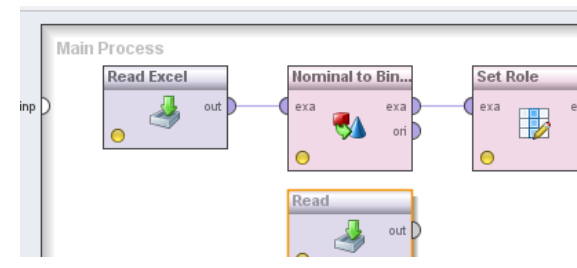

另外, 引入 Apply Association Rules 部件, 从 confidence aggregation method 中选择 binary, 根据购买

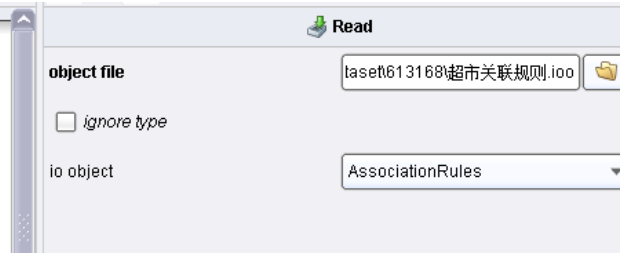

明细数据集应用关联规则集合，进行商品推荐。 


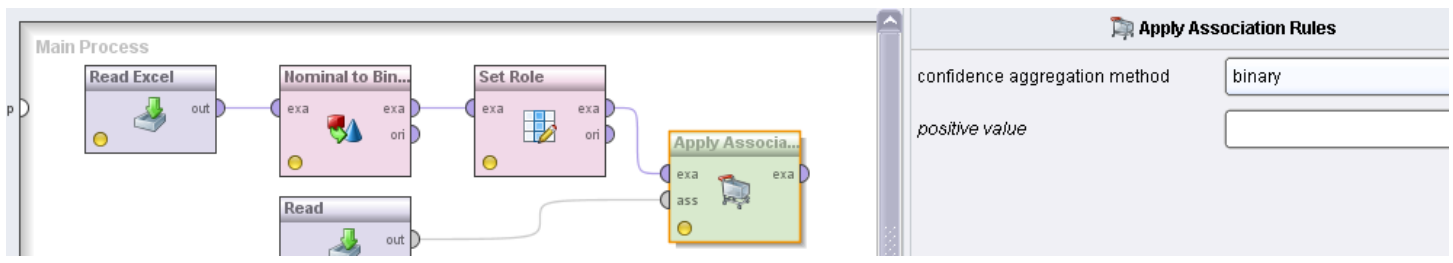

最后, 引入部件 Rename by Replacing, 1) 从 attribute filter type 中选择 all；2) 勾选 include special attributes；3）将 replace what 设成正则表达式 “confidencel(PRODUCT_(.+)l)”; 4) 将 replace by 设成
“推荐 $\$ 1 ” 。$ 该部件将形式为 “confidence(PRODUCT_XXX)”的属性名改成“推荐

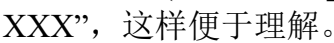

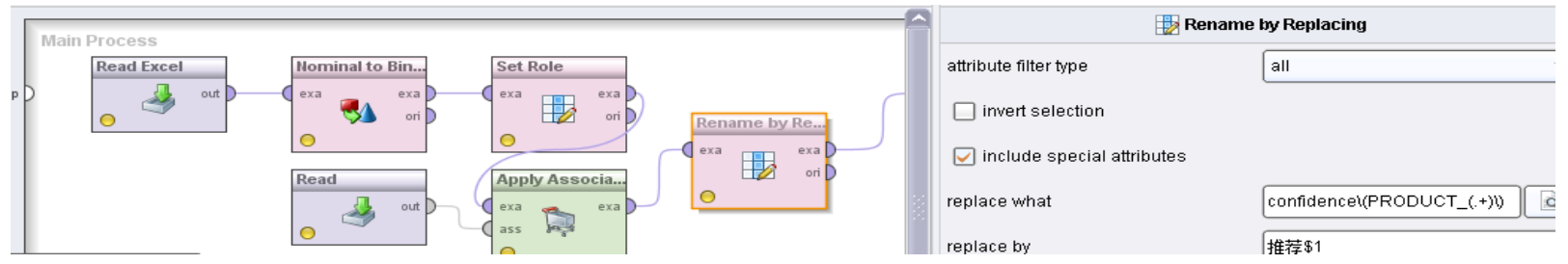

(3) 运行结果分析

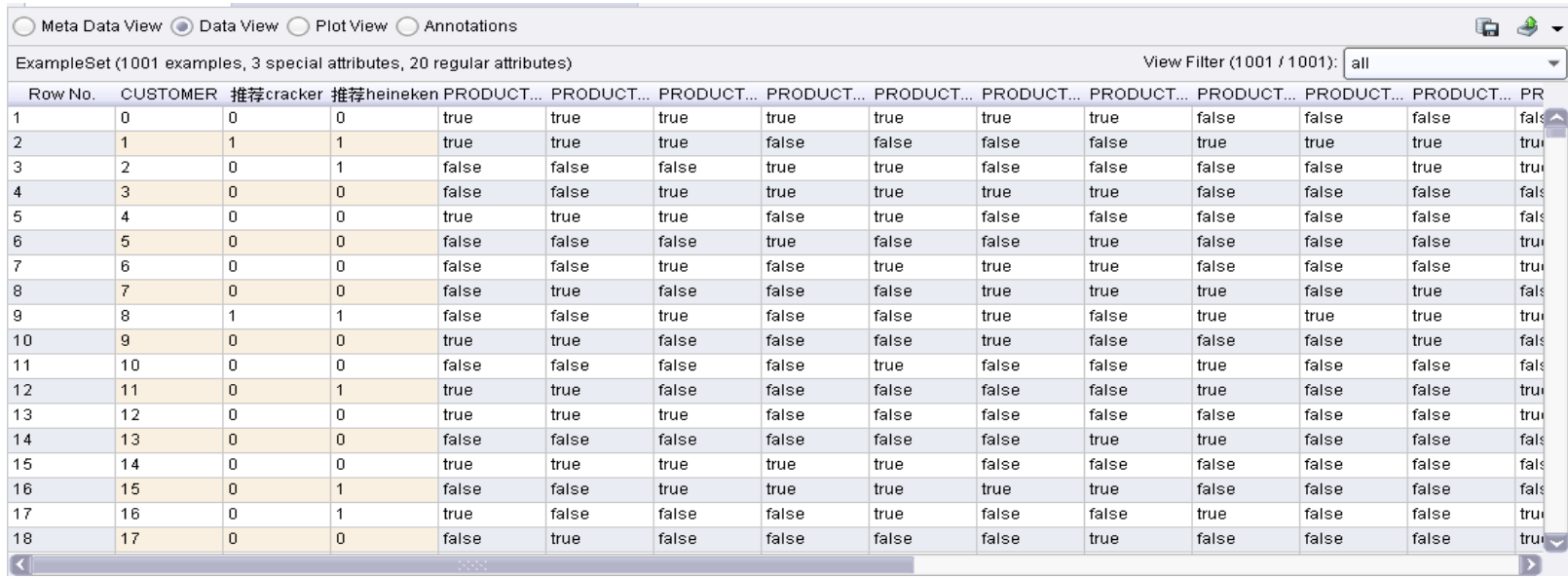

以 ROW NO.16 和 17 为例, 向顾客推荐 heineken 产 品都显示为 1 , 即可向这两个顾客推荐 heineken 产品。从 实际数据来看, 顾客 16 是没有购买 heineken, 而顾客 17 实际上是有买产品 heineken 的。因此, 关联分析, 就可 以给顾客推荐顾客潜在想购买的商品。

注意, 推荐 cracker 和推荐 heineken 全为 1 的客户, 不推荐任何商品, 因为该在实际情况下, 两样产品都购 买了。

\section{4. 总结超市如何运用数据结果}

基于顾客的购买清单, 使用 RapidMiner 工具, 我们 做出了关联分析, 并给出以下三点的建议。首先, 超市 可以把相关性强的商品, 布置在相近的地方, 达到优化 商品布局的目的。其次, 对于关联性强的商品, 可以进 行捆绑销售并对于捆绑的商品给与一定的优惠, 从而设
计促销方案。最后, 在顾客进行购物的时候, 销售人员 还可以给与顾客感兴趣的产品做快速商品推荐。通过吸 引更多的顾客, 提高顾客的光顾次数, 并且提高每个顾 客的单次的消费金额，超市才得以获取更多的利润总额 [4]。因此本文提出的超市商品捆绑销售模型具有一定的 实用意义。

\section{REFERENCES}

[1] Hongxia Cheng, Research on Data Mining Algorithms Based on Association Rules, Computer Knowledge and Technology, 2007.

[2] Ying Peng, Data Mining Overview, Journal of Dehong Teachers' College, 2009.

[3] Fang YU, The application research of association 
rules mining in supermarket marketing analysis, Harbin Institute of Technology, 2010.

[4] Fang YU, Application of Association Analysis in Supermarket Bundle Sales, Market Modernization, 2010. 